\title{
Fatal respiratory diphtheria in a visitor to Canada
}

\author{
Scott Cholewa MA BHSc DPA, Fareen Karachiwalla MD MPH, Sarah E. Wilson MD MSc, Jeya Nadarajah MD MSc, \\ Julianne V. Kus PhD MSc
}

Cite as: CMAJ 2021 January 4;193:E19-22. doi: 10.1503/cmaj.200707

A 69-year-old visitor to Canada from India presented to hospital with acute respiratory distress 48 hours after arrival. The patient reported sore throat, hoarse voice and dry cough, which had developed on departure from India and progressed over 48 hours to shortness of breath, fever and diaphoresis. The patient was otherwise healthy and had no ill contacts, but was not up to date with routinely administered vaccines.

On review in a telemedicine clinic, the patient was advised to present to the emergency department immediately, where they were noted to have a slightly swollen neck, no palpable lymph nodes and no stridor. They reported a feeling of something sticking in their throat, although obstruction was not noted. A neck radiograph showed prominent prevertebral soft tissue swelling. A chest radiograph showed left lower lobe consolidation. We diagnosed possible influenza, with concomitant bacterial pneumonia, and as per local guidelines, gave the patient ceftriaxone and oseltamivir. We admitted the patient, and over the next 48 hours, they progressively deteriorated to hypoxemic respiratory failure and acute respiratory distress syndrome, which was ultimately fatal.

Results of the following tests were negative: blood samples for culture, nasopharyngeal swab for respiratory virus culture (adenovirus, influenza A and B, human metapneumovirus and respiratory syncytial virus), and Legionella urine antigen. After the patient died, the endotracheal sputum culture we sent at the time of intubation was reported to show pure, light growth of "coryneform" bacteria. "Coryneform" or "diphtheroid" bacteria cultured from nonsterile sites are typically considered commensal flora of little clinical significance. However, given it was a pure culture, we began further investigations.

Matrix-assisted laser desorption/ionization time-of-flight mass spectrometry (MALDI-TOF MS) identified the bacteria as Corynebacterium diphtheriae. As this microorganism is rarely encountered in most Canadian laboratories, we forwarded the isolate to the provincial and national reference laboratories for identification and toxin testing. The isolate was confirmed - by polymerase chain reaction (PCR) at the National Microbiology Laboratory - to have the tox gene, which confers the ability to produce diphtheria toxin.

\section{KEY POINTS}

- Although uncommon, cases of classic, toxin-producing respiratory diphtheria still occur in Canada and should be included in the differential diagnosis for people with acute respiratory illness who have recently arrived from a diphtheriaendemic country or are inadequately protected through immunization.

- Respiratory diphtheria is a medical emergency requiring administration of antitoxin and antimicrobial therapy.

- Local public health management of diphtheria cases involves contact tracing and may require administration of postexposure prophylaxis and immunization.

- Toxigenic diphtheria is vaccine preventable. Those born in Canada, newcomers to Canada and those planning to travel to areas where diphtheria continues to circulate should ensure their immunizations are up to date.

Although final confirmation that this was a diphtheria toxinproducing strain (via the modified Elek test) was still pending, the National Microbiology Laboratory advised that the majority of tox gene-positive isolates are toxin producers. ${ }^{1}$ Given this, together with the patient's clinical history and epidemiologic information, we decided to initiate contact tracing. Diphtheria toxin production was reported 6 days after the positive PCR result.

Contact tracing was made possible through a collaboration between local public health and the hospital's infection prevention and control department, with support provided by Public Health Ontario and the Public Health Agency of Canada. Four household members were identified, along with 7 health care workers who may have been exposed to oropharyngeal secretions in the absence of personal protective equipment. All contacts were advised of the exposure; assessed for any signs and symptoms; swabbed with both a nasopharyngeal and throat swab for bacterial specimens; given antimicrobial chemoprophylaxis (erythromycin); immunized, if they were not up to date with relevant vaccine; and excluded from school and health care settings until culture results were available. Secondary transmission was ruled out among all contacts. 


\section{Discussion}

Increased global travel and growing vaccine hesitancy present opportunities for uncommon medical and public health emergencies - such as toxin-producing, respiratory diphtheria - to present in Canada. This case serves as an important reminder to clinicians to consider this diagnosis, particularly in people recently arriving from endemic areas with acute respiratory illness, and to promote vaccination across the lifespan.

\section{Epidemiology}

Diphtheria is uncommon in Canada, owing to the success of immunization programs that were introduced in the 1920 s. Nationally, the incidence of toxigenic diphtheria has declined from about 100 cases per 100000 population in 1924 to 0.03 cases per 100000 population ( $n=10$ cases) in $2017 .{ }^{2}$ The most recent fatal case in a Canadian resident occurred in 2010, and in Ontario, the last reported case of toxigenic respiratory diphtheria occurred in $1995 .^{3}$

\section{Clinical presentation and acute management}

Diphtheria is a disease with a range of clinical manifestations and types. It is classified according to both anatomic site of infection (e.g., cutaneous versus respiratory) and whether it is toxin producing or not. Respiratory diphtheria is caused by toxigenic strains of the gram-positive bacteria C. diphtheriae or, more rarely, by other toxigenic species (C. ulcerans and $C$. pseudotuberculosis). ${ }^{4}$ Initial symptoms of pharyngeal or tonsillar diphtheria typically include fever, pharyngitis, hoarse voice and enlarged cervical lymph nodes, progressing to upper airway obstruction and acute respiratory distress. ${ }^{4}$ Two classic signs of toxigenic respiratory diphtheria include a greyish-white membrane on the tonsils and significant swelling of the uvula, tonsils, cervical lymph nodes, anterior neck and submandibular area, resembling a "bull neck."

Complications of toxin dissemination include peripheral neuropathy and myocarditis. ${ }^{6}$ The estimated case fatality rate of classic respiratory diphtheria is $5 \%-10 \% .{ }^{5}$ Whether a strain is toxigenic is determined by the presence of the tox gene, ascertained

Box 1: Roles and responsibilities for toxigenic diphtheria follow-up

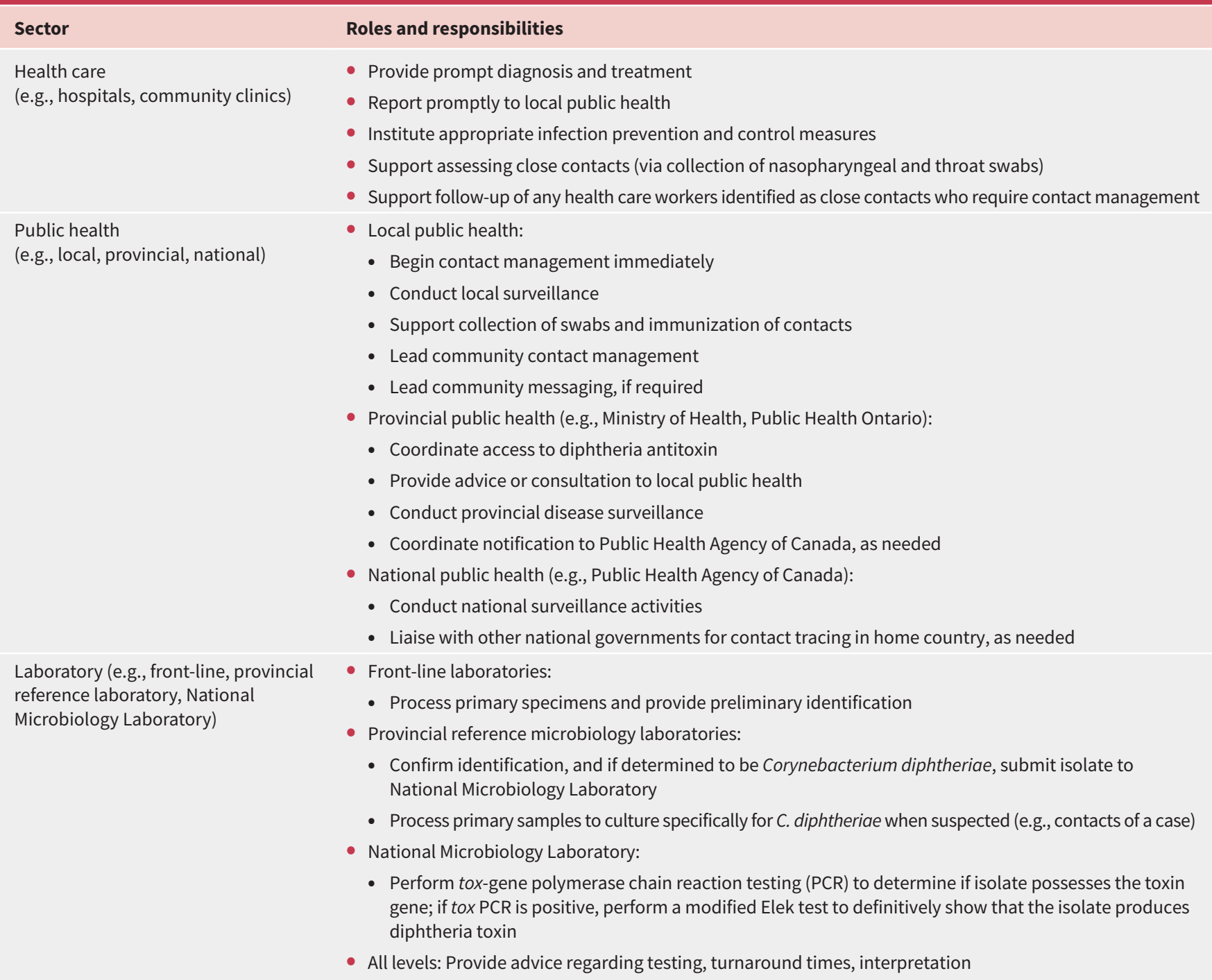


by using PCR and, if positive, confirmation that diphtheria toxin is expressed, using the modified Elek test. Toxigenic respiratory diphtheria is a medical emergency requiring both diphtheria antitoxin and antimicrobial therapy, without waiting for laboratory confirmation. Careful airway management and monitoring of cardiac and neurologic function is essential. Canadian clinicians can access diphtheria antitoxin by contacting public health departments. In Ontario, the Ministry of Health coordinates this function, with a centralized supply of diphtheria antitoxin. ${ }^{7}$

\section{Diagnosis}

Although $C$. diphtheriae isolates are relatively infrequent in Canada, changes in front-line clinical microbiology laboratories, including adoption of MALDI-TOF MS, may help to increase identification of these isolates. This is summarized in a 2019 publication that described a $1200 \%$ increase in referrals to the National Microbiology Laboratory for toxin testing between 2006 and 2019 (from 8 per yr to about 180 per yr). Most isolates were taken from cutaneous, not respiratory, sites, and only $8 \%$ were toxigenic. ${ }^{1}$ Despite increased microbiological detection, classic respiratory toxigenic diphtheria remains uncommon in Canada.

\section{Follow-up}

In Canada, the public health management of toxigenic diphtheria is critical to prevent further transmission and requires a coordinated response (Box 1), including facilitation of access to diphtheria antitoxin, contact identification and contact follow-up (i.e., assessment of immunization status, nasopharyngeal and throat swabs to rule out early evidence of secondary transmission and chemoprophylaxis). If initial swabs are positive, followup cultures taken at least 24 hours after the cessation of antimicrobial therapy are required, and an expanded ring of contact identification may need to be considered. Although public health management of close contacts is typically required only for toxigenic diphtheria, it is not uncommon for local public health authorities to receive reports of identification of $C$. diphtheriae culture several days before toxigenicity results are available. This challenges the timeliness of implementing public health actions in the event toxigenicity is confirmed. One UK guideline ${ }^{8}$ provides more specific guidance on contact tracing before toxigenicity results are available, depending on the clinical severity of the index case, epidemiologic risk factors that increase the likelihood of toxigenicity (for example, travel) and the estimated time to definitive laboratory results. Nontoxigenic diphtheria is not reportable in Canada and typically does not require contact follow-up, although clusters of nontoxigenic cutaneous diphtheria in marginalized populations in both Canada and elsewhere are well described. ${ }^{9,10}$

In our case, the timelines associated with culture identification and subsequent confirmation of toxigenicity presented public health challenges. The clinical and local public health teams received the results confirming the presence of $C$. diphtheriae and toxigenicity after a full incubation period (10 d) had passed. ${ }^{3}$ Although all close contacts were asymptomatic, contact management activities were carried out because of the possibility of an asymptomatic carrier state, a possible source of further transmission. ${ }^{8}$ Recent case reports have documented secondary transmission of cutaneous and respiratory toxigenic C. diphtheriae to close contacts, detected through swabbing before chemoprophylaxis. Close contacts are defined as household members, persons who have had close face-to-face contact to a case such as intimate contact and health care workers exposed to oropharyngeal secretions from the case, following the same principles as contact management for invasive meningococcal disease. ${ }^{11,12}$ In a case report of respiratory diphtheria, subsequent transmission from an asymptomatic carrier was identified after additional contact follow-up activities. ${ }^{11}$

Lastly, we considered how to manage close contacts associated with the international flight to Canada. Both meningococcal disease and diphtheria are associated with droplet transmission. With limited guidance in this area, we followed the approach taken in Canada for cases of invasive meningococcal disease in people who have travelled by air, which is to ensure contact tracing for passengers sitting on either side of the case on plane rides lasting more than 8 hours. ${ }^{3,8}$ However, in this specific case, the patient sat beside their spouse in the window seat, with no one on the other side, so this yielded no additional contacts. We sent formal notification about the case to India via the Public Health Agency of Canada. No other close contacts were identified in India.

This fatal case is a reminder that diphtheria, endemic in many parts of the world, can present itself at any time in Canada. Recent case reports from nonendemic countries have described both toxigenic respiratory and cutaneous diphtheria among returning travellers, visitors, immigrants and refugees. ${ }^{11,12}$ In patients presenting with respiratory distress, clinicians should consider the diagnosis of diphtheria, particularly among recent travellers or immigrants from endemic areas. Immunization status of newcomers and frequent travellers should be assessed to ensure individuals are up to date. In a time of increasing vaccine hesitancy and extensive global movement, presentations of longforgotten diseases such as diphtheria cannot be ignored.

\section{References}

1. Bernard KA, Pacheco AL, Burdz T, et al. Increase in detection of corynebacterium diphtheriae in Canada: 2006-2019. Can Commun Dis Rep 2019;45:296-301. Available: www.canada.ca/content/dam/phac-aspc/documents/services/reports -publications/canada-communicable-disease-report-ccdr/monthly-issue/2019 -45/issue-11-november-7-2019/ccdrv45i11a04-eng.pdf (accessed 2019 Dec. 14).

2. Reported cases from 1924 to 2018 in Canada - notifiable diseases on-line [data file]. Ottawa: Public Health Agency of Canada; modified 2020 Aug. 11. Available: https://diseases.canada.ca/notifiable/charts?c=pl (accessed 2019 Dec. 15).

3. Infectious diseases protocol - Appendix A: Disease-specific chapters. Chapter: Diphtheria. Toronto: Ministry of Health and Long-Term Care; 2019:1-13. Available: http://health.gov.on.ca/en/pro/programs/publichealth/oph_standards/ docs/diphtheria_chapter.pdf (accessed 2019 Dec. 15).

4. Heymann DL, editor. Control of communicable diseases manual. 20th ed. Washington (DC): American Public Health Association; 2014.

5. Diphtheria: for health professionals. Ottawa: Public Health Agency of Canada; modified 2018 June 21. Available: www.canada.ca/en/public-health/services/ immunization/vaccine-preventable-diseases/diphtheria/health-professionals. html (accessed 2019 Dec. 7).

6. Skogmar S, Tham J. Severe diphtheria with neurologic and myocardial involvement in a Swedish patient: a case report. BMC Infect Dis 2018;18: 359. 
7. Diphtheria guide for health care professionals. Toronto: Public Health Division, Ministry of Health and Long-Term Care; 2015. Available: www.health.gov.on. ca/en/pro/publications/disease/pdf/2015-05-22-diphtheria-guide-health-care -professionals.pdf (accessed 2019 Dec. 15).

8. Diphtheria Guidelines Working Group. Public health control and management of diphtheria (in England and Wales): 2015 guidelines. London (UK): Public Health England; 2015:1-47. Available: https://assets.publishing.service.gov.uk/ government/uploads/system/uploads/attachment_data/file/774753/Diphtheria _Guidelines_Final.pdf (accessed 2019 Dec. 15).

9. Lowe CF, Bernard KA, Romney MG. Cutaneous diphtheria in the urban poor population of Vancouver, British Columbia, Canada: a 10-year review. J Clin Microbiol 2011;49:2664-6.
10. Dangel A, Berger A, Konrad R, et al. Geographically diverse clusters of nontoxigenic infection, German, 2016-2017. Emerg Infect Dis 2018;24:1239-45.

11. Edwards D, Kent D, Lester C, et al. Transmission of toxigenic Corynebacterium diphtheriae by a fully immunised resident returning from a visit to West Africa, United Kingdom, 2017. Euro Surveill 2018;23:1700681.

12. Jané M, Vidal MJ, Camps N, et al. A case of respiratory toxigenic diphtheria: contact tracing results and considerations following a 30-year disease-free interval, Catalonia, Spain, 2015. Euro Surveill 2018;23:17-00183.

\section{Competing interests: None declared.}

This article has been peer reviewed.

The authors have obtained consent from the patient's next of kin.

Affiliations: Public Health Branch (Cholewa, Karachiwalla), Regional Municipality of York, Newmarket, Ont.; Dalla Lana School of Public Health (Karachiwalla, Wilson), and Faculty of Medicine (Nadarajah), and Department of Laboratory Medicine and Pathobiology (Kus), University of Toronto; Public Health Ontario (Wilson, Kus), Toronto, Ont.; Department of Family Medicine, School of Medicine (Karachiwalla), Queen's University, Kingston, Ont.; Institute for Clinical Evaluative Studies, (Wilson), Toronto, Ont.

Contributors: Scott Cholewa and Fareen Karachiwalla contributed to the conception and design of the work. All of the authors contributed to drafting the manuscript. All of the authors revised it critically for important intellectual content, gave final approval of the version to be published and agreed to be accountable for all aspects of the work.
Funding: Funding for this case investigation and public health follow up was from existing organizational operating budgets.

Content licence: This is an Open Access article distributed in accordance with the terms of the Creative Commons Attribution (CC BY-NC-ND 4.0) licence, which permits use, distribution and reproduction in any medium, provided that the original publication is properly cited, the use is noncommercial (i.e., research or educational use), and no modifications or adaptations are made. See: https://creativecommons.org/licenses/by-nc-nd/4.0/

Acknowledgements: The authors acknowledge the contributions of the following individuals: Dr. Michelle Murti, Public Health Ontario; Martina Cuillerier, Thomas Lo, Andrea Main, Marjolyn Pritchard and Candace Wong, York Region Public Health; Dr. Deborah Yamamura, Hamilton Health Sciences Centre; Kathryn Bernard, National Microbiology Laboratory; and laboratory staff at Hamilton Health Sciences Centre, Public Health Ontario and the National Microbiology Laboratory.

Correspondence to: Scott Cholewa, scott.cholewa@york.ca 